\title{
Neural Networks Approximator Based Robust Adaptive Controller Design of Hypersonic Flight Vehicles Systems Coupled with Stochastic Disturbance and Dynamic Uncertainties
}

\author{
Guoqiang Zhu, Lingfang Sun, and Xiuyu Zhang \\ School of Automation Engineering, Northeast Electric Power University, Jilin 132012, China \\ Correspondence should be addressed to Xiuyu Zhang; zhangxiuyu80@163.com
}

Received 9 March 2017; Accepted 8 August 2017; Published 18 September 2017

Academic Editor: Weihai Zhang

Copyright (C) 2017 Guoqiang Zhu et al. This is an open access article distributed under the Creative Commons Attribution License, which permits unrestricted use, distribution, and reproduction in any medium, provided the original work is properly cited.

\begin{abstract}
A neural network robust control is proposed for a class of generic hypersonic flight vehicles with uncertain dynamics and stochastic disturbance. Compared with the present schemes of dealing with dynamic uncertainties and stochastic disturbance, the outstanding feature of the proposed scheme is that only one parameter needs to be estimated at each design step, so that the computational burden can be greatly reduced and the designed controller is much simpler. Moreover, by introducing a performance function in controller design, the prespecified transient and performance of tracking error can be guaranteed. It is proved that all signals of closed-loop system are uniformly ultimately bounded. The simulation results are carried out to illustrate effectiveness of the proposed control algorithm.
\end{abstract}

\section{Introduction}

It is well known that stochastic disturbances appear often in many practical systems $[1,2]$. Their existence is a source of instability of the control systems; thus, the investigations on stochastic systems have received considerable attention in recent years. Several classes of nonlinear systems with stochastic disturbances and dynamic uncertainties were stabilized by using adaptive neural networks based control. In [3] a backstepping control scheme was proposed for the stochastic nonlinear strict-feedback system.

A framework based on the stochastic Liouville Equation (SLE) was provided for both three-state and six-state Vinh's equations for hypersonic entry in Mars atmosphere [4]. Wu et al. [5] applied the stochastic small-gain theorem and backstepping design technique in the stochastic nonlinear systems with uncertain nonlinear functions and unmodeled dynamics.

In recent years, hypersonic flight vehicles (HFVs) have received a great deal of attention around the world, which offer a promising technology for cost-efficient and reliable access to space and are especially suitable for prompt global response [6-8]. However, the design of control systems for HFVs is a challenging work due to the longitudinal dynamics of HFVs being highly nonlinear and strong couplings between the propulsive and aerodynamic forces [9-11].

The modeling inaccuracy, parameters uncertainties, and external disturbances can result in strong adverse effects on the performance of HFVs control systems. As a result, the onboard flight control systems design of HFVs presents numerous challenges.

Based on modern control techniques, various flight control systems have been designed to the longitudinal dynamics of HFVs. In [12], the adaptive backstepping method was used to design controllers for HFVs, while fuzzy logic systems (FLSs) and neural networks (NNs) were used to approximate the unknown system dynamics in $[13,14]$. However, backstepping design suffers from the problem of "explosion of complexity" caused by the repeated differentiations of nonlinear functions [5, 15-17]. To eliminate this problem, dynamic surface control (DSC) was applied to longitudinal dynamics of HFVs in [18-23], which uses a low-pass filter at each design 
step to avoid the derivatives of nonlinear functions. In the above control schemes, the uncertain nonlinear functions in the HFVs model were approximated by NNs or FLSs using their universal approximation capability [24-26]. However, the drawback of these control schemes is that the number of adaptation laws depends on the number of the NNs nodes or the number of the fuzzy rules. With an increase of NNs nodes or fuzzy rules, the number of estimated parameters will increase significantly. To solve this problem, in [2730 ], the norm of the ideal weighting vector in NNs or FLSs was considered as the estimation parameter instead of the elements of weighting vector. Thus, the number of adaptation laws is reduced considerably.

Besides, an interesting question raised by DSC or backstepping control schemes is the output error transient performance. Recently, to guarantee a prespecified tracking performance, a backstepping design based on NNs was proposed for a class of uncertain nonlinear systems, and it is shown that the tracking errors can converge to predefined arbitrarily small residue sets with prescribed convergence rate and maximum overshoot $[31,32]$. However, to our best knowledge, limited attention has been paid to this problem for the controller design of HFVs.

Another feature of the proposed scheme is that the radial basis function (RBF) NNs are employed to compensate for the uncertain nonlinear functions. By using the minimal learning technique, only one parameter needs to be update online at each design step regardless of the NNs input-output dimension and the number of NNs nodes. As a result, the number of adaptation laws and the computational burden are greatly reduced.

Inspired by the aforementioned discussions, in this paper, we divide the control oriented model (COM) of HFVs into two parts: velocity subsystem and altitude subsystem. Dynamic inversion method is employed to design the controller for velocity subsystem, while DSC strategy is used for altitude subsystem. Besides, a performance function is introduced to obtain a virtual error constraint variable, which can ensure the prescribed transient performance. By this transformation, the original tracking error can converge to predefined arbitrarily small residue sets with prescribed convergence rate and maximum overshoot. Simulation results are presented to demonstrate the efficiency of the proposed scheme.

The rest of this paper is organized as follows. In Section 2, the nonlinear longitudinal dynamics model of HFVs is presented. The controllers design and the stability analysis are given in Section 3. The simulation results are illustrated in Section 4, followed by conclusion of this paper in Section 5.

\section{Problem Formulation and Preliminaries}

The control oriented model (COM) of the longitudinal dynamics of HFV considered in this study is taken form $[8,18]$. The equations of the COM model are expressed as

$$
\begin{aligned}
& \dot{V}=\frac{(T \cos \alpha-D)}{m}-g \sin \gamma+\omega_{1}, \\
& \dot{h}=V \sin \gamma,
\end{aligned}
$$

$$
\begin{aligned}
& \dot{\gamma}=\frac{(L+T \sin \alpha)}{m V}-\frac{g \cos (\gamma)}{V}, \\
& \dot{\alpha}=q-\dot{\gamma}+\omega, \\
& \dot{q}=\frac{M_{y y}}{I_{y y}}+\omega_{5},
\end{aligned}
$$

where $V$ is the velocity, $\gamma$ is the flight path angle, $h$ is the altitude, $\alpha$ is the attack angle, $q$ is the pitch rate, and $\omega_{i}, i=1,5$, are the uncertain external disturbance. $T(V, \beta)$, $D(V, \alpha), L(V, \alpha)$, and $M_{y y}\left(V, \alpha, q, \delta_{E}\right)$ represent the thrust, drag, lift-force, and pitching moment, respectively, which can be expressed as

$$
\begin{aligned}
T & =C_{T}^{\alpha^{3}} \alpha^{3}+C_{T}^{\alpha^{2}} \alpha^{2}+C_{T}^{\alpha} \alpha+C_{T}^{0}, \\
D & =\bar{q} S C_{D}\left(\alpha, \delta_{e}\right), \\
L & =\bar{q} S C_{L}\left(\alpha, \delta_{e}\right), \\
M_{y y} & =z_{T} T+\bar{q} S \bar{c}\left[C_{M, \alpha}(\alpha)+C_{M, \delta_{e}}\left(\delta_{e}\right)\right],
\end{aligned}
$$

with $C_{L}=C_{L}^{\alpha} \alpha+C_{L}^{0}, C_{D}=C_{D}^{\alpha^{2}} \alpha^{2}+C_{D}^{\alpha} \alpha+C_{D}^{0}, C_{M, \alpha}(\alpha)=$ $C_{M, \alpha}^{\alpha^{2}} \alpha^{2}+C_{M, \alpha}^{\alpha} \alpha+C_{M, \alpha}^{0}, C_{T}^{\alpha^{3}}=\beta_{1} \Phi+\beta_{2}, C_{T}^{\alpha^{2}}=\beta_{3} \Phi+\beta_{4}, C_{T}^{\alpha}=$ $\beta_{5} \Phi+\beta_{6}, C_{T}^{0}=\beta_{7} \Phi+\beta_{8}, \bar{q}=\rho V^{2} / 2$, and $\rho=\rho_{0} \exp (-(h-$ $\left.h_{0}\right) / h_{s}$ ), where $\delta_{e}$ is the elevator deflection and $\Phi$ is the throttle setting. Letting $\theta$ denote the pitch angle, we have $\theta=\alpha+\gamma$. Then, we define state variables as $x=\left[x_{1}, x_{2}, x_{3}, x_{4}, x_{5}\right]^{T}$, with $x_{1}=V, x_{2}=h, x_{3}=\gamma, x_{4}=\theta$, and $x_{5}=q$. Note that the flight path angle $\gamma$ is typically very small during the trimmed cruise condition, which justify the approximation $\sin (\gamma) \approx \gamma$, so the system (1) can be rewritten as

$$
\begin{aligned}
& \dot{x}=f(x)+g(x) u+\omega, \\
& y=\left[x_{1}, x_{2}\right],
\end{aligned}
$$

where $f(x)=\left[f_{1}(x), f_{2}(x), f_{3}(x), f_{4}(x), f_{5}(x)\right]^{T}, g(x)=$ $\left[g_{1}(x), g_{2}(x), g_{3}(x), g_{4}(x), g_{5}(x)\right]^{T}, f_{1}(x)=\left(\beta_{a} \varphi_{0} \cos \alpha-\right.$ $D) / m-g \sin \gamma, f_{2}(x)=0, f_{3}=\bar{q} S\left(C_{L}^{0}-C_{L}^{\alpha} x_{2}\right) / m V+$ $T \sin \alpha / m V-g \cos x_{2} / V, f_{4}(x)=0, f_{5}=\left(z_{T} T+\right.$ $\left.\bar{q} S \bar{c} C_{M, \alpha}(\alpha)\right) / I_{y y}, g_{1}(x)=\beta_{b} \varphi_{0} \cos \alpha / m, g_{2}(x)=V, g_{3}(x)=$ $\bar{q} S C_{L}^{\alpha} / m V, g_{4}(x)=1, g_{5}(x)=\bar{q} S \bar{c} c_{e} / I_{y y}, \beta_{a}=\left[\beta_{2}, \beta_{4}, \beta_{6}, \beta_{8}\right]$, $\beta_{b}=\left[\beta_{1}, \beta_{3}, \beta_{5}, \beta_{7}\right]$, and $\varphi_{0}=\left[\alpha^{3}, \alpha^{2}, \alpha, 1\right]^{T}$. Since the values of the inertial and the aerodynamic parameters are uncertain, the aforementioned $f_{i}(x)$ and $g_{i}(x), i=1, \ldots, 5$, are unknown functions. Moreover, as stated in [18], from the model of the HFV, it is easy to check that $g_{1}(x), g_{2}(x)$, and $g_{3}(x)$ are always strictly positive and $g_{5}(x)$ is strictly negative since $c_{e}$ is negative. With these observations in mind, we have the following assumption.

Assumption 1. Notice that there exist positive constants $b_{i m}$ and $b_{i M}$ such that $0<b_{i m} \leq\left|g_{i}(x)\right| \leq b_{i M}, i=1, \ldots, 5$.

Assumption 2. $V_{d}$ and its first derivative are known and bounded, while $h_{d}$ and its first two derivatives are known and bounded. 
Remark 3. In the following proposed scheme, both $b_{i m}$ and $b_{i M}$ do not appear in the final control law and are used only for stability analysis, so they can be unknown.

Remark 4. The COM model of HFV is an MIMO system that has cross-channel coupling. In order to retain simplicity, we divide the model into velocity and altitude subsystems, the coupling effect is treated as a part of unknown nonlinear functions, and then RBF NNs are used to approximate them.

2.1. Radial Basis Function Neural Network Approximation. In this study, the radial basis function neural networks (RBF NNs) are used to approximate the continuous unknown functions on a given compact set. Mathematically, an RBF NN form [26] can be expressed as

$$
F(\xi)=W^{T} \psi(\xi)
$$

where $F \in \mathbb{R}$ and $\xi \in \mathbb{R}^{n}$ are the NN output and input, $W \in \mathbb{R}^{n}$ is an $N$-dimensional weight vector, and $\psi(\xi)=\left[\psi_{1}(\xi), \ldots, \psi_{N}(\xi)\right]^{T}$ is the Gaussian function with the following form:

$$
\begin{aligned}
\psi_{i}(\xi)=\frac{1}{\sqrt{2 \pi} \phi} \exp \left(-\frac{\left\|\xi-\xi_{i}\right\|^{2}}{2 \phi^{2}}\right) & \\
& \phi>0, i=0, \ldots, N, N
\end{aligned}
$$

where $\xi_{i} \in \mathbb{R}^{n}$ is a constant called the center of the Gaussian function and $\phi \in \mathbb{R}$ is the width, respectively.

Lemma 5. According to the approximation property of $R B F$ NNs, given any continuous function $F(\xi): \Omega_{\xi} \rightarrow \mathbb{R}$ with $\Omega_{\xi} \subset \mathbb{R}^{n}$ a compact set, and any constant $\epsilon>0$, by appropriately choosing $\phi$ and $\xi_{i}, i=1, \ldots, N$, for some sufficiently large integer $N$, there exists an optimal weight $W^{* T}$ such that $W^{* T} \psi(\xi)$ can approximate the given function $F(\xi)$ with approximation error $\Delta(\xi)$ bounded by $\epsilon$ [26].

$$
F(\xi)=W^{* T} \psi(\xi)+\Delta(\xi), \quad|\Delta(\xi)| \leq \epsilon, \forall \xi \in \Omega_{\xi},
$$

since $W^{*}$ is unknown; we need to estimate it online.

\section{Adaptive Controller Design and Stability Analysis}

3.1. Performance Functions and Error Transformation. Similar to $[31,32]$, the mathematical expression of the prescribed tracking performance is given by

$$
-m_{i} p_{i}(t)<e_{i}(t)<n_{i} p_{i}(t)
$$

where $e_{i}=y_{i}-y_{i d}, i=1,2$, are the tracking error, $m_{i}$ and $n_{i}$ are given positive constants, and the performance function $p_{i}$ is defined as smooth and decreasing positive function. From (7), one can see that $-m_{i} p_{i}(0)$ and $n_{i} p_{i}(0)$ are the lower and upper bound of the undershoot of $e_{i}(t)$, respectively. $m_{i} p_{i}(\infty)$ and $n_{i} p_{i}(\infty)$ represent the maximum allowable size of the steady-state value of $e_{i}(t)$.
To transform (7) into an equivalent unconstrained one, define the error transformation function $\Upsilon_{i}\left(S_{i}\right)=e_{i}(t) / p_{i}(t)$, where $S_{i}$ is the transformed error and $\Upsilon_{i}\left(S_{i}\right)$ is a smooth, strictly increasing function having the following properties:

$$
\begin{aligned}
& \lim _{S \rightarrow-\infty} \Upsilon_{i}\left(S_{i}\right)=-m_{i}, \\
& \lim _{S \rightarrow+\infty} \Upsilon_{i}\left(S_{i}\right)=n_{i} .
\end{aligned}
$$

From (8), if $S_{i}$ is bounded, we have $-m_{i}<\Upsilon_{i}\left(S_{i}\right)<n_{i}$, and thus (7) holds. Hence, to achieve the prespecified tracking performance, one only needs to keep $S_{i}$ bounded. The inverse transformation of $\Upsilon_{i}\left(S_{i}\right)$ can be expressed as

$$
S_{i}=\Upsilon_{i}^{-1}\left(\frac{e_{i}(t)}{p_{i}(t)}\right):=\Theta_{i}\left(\frac{e_{i}(t)}{p_{i}(t)}\right),
$$

and differentiating (9) yields

$$
\dot{S}_{i}=\eta_{i} \dot{y}_{i}-\eta_{i} v_{i}
$$

From the properties of the transformation, it is clear that $\eta_{i}$ and $v_{i}$ are bounded and $0<\eta_{i} \leq \leq \eta_{i}$.

3.2. Velocity Controller Design via Dynamic Inversion. In this paper, by functional decomposition, the dynamics of HFVs is decoupled into velocity and altitude subsystem. Velocity subsystem of HFV (1) can be rewritten as follows:

$$
\begin{aligned}
& \dot{x}_{1}=f_{1}(x)+g_{1}(x) \Phi+\omega_{1}, \\
& y_{1}=x_{1},
\end{aligned}
$$

where $f_{1}(x)$ and $g_{1}(x)$ are unknown nonlinear function and $g_{1} \geq b_{1 m}>0$. Then define the velocity tracking error as $e_{1}=$ $x_{1}-x_{1 d}$. According to (10) and (11) we obtain

$$
\dot{S}_{1}=-\eta_{1} v_{1}+\eta_{1} f_{1}(x)+\eta_{1} g_{1}(x) \Phi+\omega_{1} .
$$

Let $F_{1}\left(\xi_{1}\right)=(1 / 2) S_{1}-\eta_{1} v_{1}+\eta_{1} f_{1}(x)+\omega_{1}$ and $\xi_{1}:=$ $\left[x, \dot{x}_{1 d}, p_{1}, \omega_{1}\right]^{T} \in \Omega_{\xi_{1}} \subseteq \mathbb{R}^{8}$. The transformed system dynamics of (12) can be rewritten as

$$
\dot{S}_{1}=\eta_{1} g_{1}(x) \Phi-\frac{1}{2} S_{1}+F_{1}\left(\xi_{1}\right) .
$$

Since $F_{1}\left(\xi_{1}\right)$ is an unknown nonlinear function, we use an RBF NN to approximate it. Then by using Lemma 5, we have

$$
F_{1}\left(\xi_{1}\right)=W_{1}^{* T} \psi_{1}\left(\xi_{1}\right)+\Delta_{1}\left(\xi_{1}\right), \quad\left|\Delta_{1}\left(\xi_{1}\right)\right| \leq \epsilon_{1} .
$$

Choose the control signal and the adaptive update law as follows:

$$
\begin{gathered}
\Phi=-c_{1} S_{1}-\frac{1}{2} S_{1} \widehat{\vartheta}_{1} \psi_{1}^{T}\left(\xi_{1}\right) \psi_{1}\left(\xi_{1}\right), \\
\dot{\widehat{\vartheta}}_{1}=\frac{\lambda_{1}}{2} S_{1}^{2} \psi_{1}^{T}\left(\xi_{1}\right) \psi_{1}\left(\xi_{1}\right)-\lambda_{1} \sigma_{1} \widehat{\vartheta}_{1},
\end{gathered}
$$


where $\widehat{\vartheta}_{1}$ is the estimate of $\vartheta_{1}=\left\|W_{1}^{*}\right\|^{2} / \eta_{10} b_{1 m}$ and $c_{1}, \lambda_{1}$, and $\sigma_{1}$ are designed positive parameters. Consider the Lyapunov function

$$
\Gamma_{1}=\frac{1}{2} S_{1}^{2}+\frac{\eta_{10} b_{1 m}}{2 \lambda_{1}} \widetilde{\vartheta}_{1}^{2}
$$
follows.

The differential of Lyapunov function $\Gamma_{1}$ can be found as

$$
\begin{aligned}
\dot{\Gamma}_{1} \leq & S_{1} \eta_{V 0} b_{1 m} \Phi+\frac{\eta_{10} b_{1 m}}{2} S_{1}^{2} \widehat{\vartheta}_{1}^{T}\left(\xi_{1}\right) \psi_{1}\left(\xi_{1}\right) \\
& +\frac{\eta_{10} b_{1 m}}{\lambda_{1}} \widetilde{\vartheta}_{1}\left(\dot{\widehat{\vartheta}}_{1}-\frac{\lambda_{1}}{2} S_{1}^{2} \psi_{1}^{T}\left(\xi_{1}\right) \psi_{1}\left(\xi_{1}\right)\right)+\frac{1}{2} \\
& +\frac{1}{2} \epsilon_{1}^{2} .
\end{aligned}
$$

Substituting (15) into (17) yields

$$
\dot{\Gamma}_{1} \leq-c_{1} \eta_{10} b_{1 m} S_{1}^{2}-\eta_{10} b_{1 m} \sigma_{1} \widetilde{\vartheta}_{1} \widehat{\vartheta}_{1}+\frac{1}{2}+\frac{1}{2} \epsilon_{1}^{2} .
$$

According to Assumption 1 and the inequality $-\widetilde{\vartheta}_{1} \widehat{\vartheta}_{1} \leq$ $-(1 / 2) \widetilde{\vartheta}_{1}^{2}+(1 / 2) \vartheta^{2}$, we have

$$
\begin{aligned}
\dot{\Gamma}_{1} \leq & -c_{1} \eta_{10} b_{1 m} S_{1}^{2}-\frac{1}{2} \eta_{10} b_{1 m} \sigma_{1} \widetilde{\vartheta}_{1}^{2}+\frac{1}{2} \eta_{10} b_{1 m} \sigma_{1} \vartheta^{2}+\frac{1}{2} \\
& +\frac{1}{2} \epsilon_{1}^{2}
\end{aligned}
$$

$\dot{\Gamma}_{1} \leq-2 \kappa_{1} \Gamma_{1}+C_{1}$,

where

$$
\begin{aligned}
& \kappa_{1}=\min \left(c_{1} \eta_{10} b_{1 m}, \frac{1}{2} \lambda_{1} \sigma_{1}\right), \\
& \mu_{1}=\frac{1}{2} \eta_{10} b_{1 m} \sigma_{1} \vartheta^{2}+\frac{1}{2}+\frac{1}{2} \epsilon_{1}^{2} .
\end{aligned}
$$

Solving (19) gives

$$
0 \leq \Gamma_{1}(t) \leq \frac{C_{1}}{2 \kappa_{1}}+\left(\Gamma_{1}(0)-\frac{C_{1}}{2 \kappa_{1}}\right) e^{-2 \kappa_{1} t}, \quad t \geq 0,
$$

from which it is clear that, by properly choosing the design parameters $c_{1}, \lambda_{1}$, and $\sigma_{1}, S_{1}, \widehat{\vartheta}_{1}$, and $\widetilde{\vartheta}_{1}$ in the closed-loop system are uniformly ultimately bounded, and the prescribed tracking performance is guaranteed.

3.3. Attitude Controller Design via DSC. In this section, the DSC technique will be introduced to the system described by (1); the recursive design procedure contains 4 steps. In Steps $1-3$, the virtual control law is designed at each step; finally an overall control law $\delta_{e}$ is constructed at Step 4. After the error transformation (7)-(10), the altitude subsystem (1) is equivalent to

$$
\begin{aligned}
& \dot{S}_{2}=\eta_{2} g_{2}(x) x_{3}-\eta_{2} v_{2}, \\
& \dot{x}_{3}=g_{3}(x) x_{4}+f_{3}(x), \\
& \dot{x}_{4}=g_{4}(x) x_{5}+f_{4}(x), \\
& \dot{x}_{5}=g_{5}(x) \delta_{e}+f_{5}(x)+\omega_{5}, \\
& y_{2}=x_{2} .
\end{aligned}
$$

The stabilization of the transformed system (22) is sufficient to guarantee the prescribed tracking performance of altitude subsystem.

Step 1. Let $S_{2}$ given by (22) be the first error variable. Define $u_{3}$ as the first virtual control signal. Then the derivative of $S_{2}$ can be expressed as

$$
\dot{S}_{2}=\eta_{2} g_{2}(x) x_{3}-\frac{1}{2} S_{2}+F_{2}\left(\xi_{2}\right),
$$

where $F_{2}\left(\xi_{2}\right)=(1 / 2) S_{2}-\eta_{2} v_{2}$. Since $F_{2}\left(\xi_{2}\right)$ is unknown, we employ an RBF NN to approximate it on a compact set $\Omega_{\xi_{2}}$. By properly choosing the basis function vectors we have

$$
F_{2}\left(\xi_{2}\right)=W_{2}^{* T} \psi_{2}\left(\xi_{2}\right)+\Delta_{2}\left(\xi_{2}\right), \quad\left|\Delta_{2}\left(\xi_{2}\right)\right| \leq \epsilon_{2},
$$

where $\xi_{2}:=\left[x_{2}, x_{2 d}, \dot{x}_{2 d}, p_{2}\right]^{T} \in \Omega_{\xi_{2}} \subseteq \mathbb{R}^{4}$ and $\epsilon_{2}$ is a positive constant. With respect to the unknown optimal weight vector in (24), define

$$
\vartheta_{2}=\frac{\left\|W_{2}^{*}\right\|^{2}}{\eta_{20} b_{2 m}},
$$

where $b_{2 m}$ are given by Assumption 1 . Since $\vartheta_{2}$ is unknown, let $\widehat{\vartheta}_{2}$ be the estimation of $\vartheta_{2}$ and $\widetilde{\vartheta}_{2}:=\widehat{\vartheta}_{2}-\vartheta_{2}$. Consider the first Lyapunov function

$$
\Gamma_{2}=\frac{1}{2} S_{2}^{2}+\frac{\eta_{20} b_{2 m}}{2 \lambda_{2}} \widetilde{\vartheta}_{2}^{2} .
$$

The derivation of (26) can be found as follows:

$$
\begin{aligned}
\dot{\Gamma}_{2}= & S_{2} \eta_{2} g_{2}(x)\left(x_{3}-u_{3}\right)+S_{2} \eta_{2} g_{2}(x) u_{3}-\frac{1}{2} S_{2}^{2} \\
& +S_{2} W_{2}^{* T} \psi_{2}\left(\xi_{2}\right)+S_{2} \Delta_{2}\left(\xi_{2}\right)+\frac{\eta_{20} b_{2 m}}{\lambda_{2}} \widetilde{\vartheta}_{2} \dot{\hat{\vartheta}}_{2} .
\end{aligned}
$$

Using Young's inequality, it can be verified that

$$
\begin{aligned}
S_{2} W_{2}^{* T} \psi_{2}\left(\xi_{2}\right) & \leq \frac{1}{2} S_{2}^{2}\left\|W_{2}^{*}\right\|^{2} \psi_{2}^{T}\left(\xi_{2}\right) \psi_{2}\left(\xi_{2}\right)+\frac{1}{2} \\
& \leq \frac{\eta_{20} b_{2 m}}{2} \vartheta_{2} S_{2}^{2} \psi_{2}^{T}\left(\xi_{2}\right) \psi_{2}\left(\xi_{2}\right)+\frac{1}{2}, \\
S_{2} \Delta_{2}\left(\xi_{2}\right) & \leq \frac{1}{2} S_{2}^{2}+\frac{1}{2} \epsilon_{2}^{2} .
\end{aligned}
$$


Thus, (27) can be rewritten as

$$
\begin{aligned}
\dot{\Gamma}_{2} \leq & S_{2} \eta_{2} g_{2}\left(x_{3}-u_{3}\right)+S_{2} \eta_{2} g_{2} u_{3} \\
& +\frac{\eta_{20} b_{2 m}}{2} \widehat{\vartheta}_{2} S_{2}^{2} \psi_{2}^{T}\left(\xi_{2}\right) \psi_{2}\left(\xi_{2}\right)+\frac{1}{2}+\frac{1}{2} \epsilon_{2}^{2} \\
& +\frac{\eta_{20} b_{2 m}}{\lambda_{2}} \widetilde{\vartheta}_{2}\left(\dot{\hat{\vartheta}}_{2}-\frac{\lambda_{2}}{2} S_{2}^{2} \psi_{2}^{T}\left(\xi_{2}\right) \psi_{2}\left(\xi_{2}\right)\right),
\end{aligned}
$$

which suggests that we choose the virtual control signal $u_{3}$ as

$$
u_{3}=-c_{2} S_{2}-\frac{1}{2} \widehat{\vartheta}_{2} S_{2} \psi_{2}^{T}\left(\xi_{2}\right) \psi_{2}\left(\xi_{2}\right),
$$

and the adaptation law

$$
\dot{\widehat{\vartheta}}_{2}=\frac{\lambda_{2}}{2} S_{2}^{2} \psi_{2}^{T}\left(\xi_{2}\right) \psi_{2}\left(\xi_{2}\right)-\lambda_{2} \sigma_{2} \widehat{\vartheta}_{2}, \quad \widehat{\vartheta}_{2}(0) \geq 0,
$$

where $c_{2}, \lambda_{2}$, and $\sigma_{2}$ are positive design parameters. Then substituting (30) and (31) into (29), we get

$$
\begin{aligned}
\dot{\Gamma}_{2} \leq & -\eta_{20} b_{2 m} c_{2} S_{2}^{2}+S_{2} \eta_{2} g_{2}\left(x_{3}-u_{3}\right)-\eta_{20} b_{2 m} \sigma_{2} \widetilde{\vartheta}_{2} \widehat{\vartheta}_{2} \\
& +\frac{1}{2}+\frac{1}{2} \epsilon_{2}^{2} .
\end{aligned}
$$

Introduce a new state variable $x_{3 d}$, which can be obtained by the following first-order filter:

$$
\tau_{3} \dot{x}_{3 d}+x_{3 d}=u_{3}, \quad x_{3 d}(0)=u_{3}(0) .
$$

Step $j(j=2,3)$. Define the $i$ th surface error $S_{i}=x_{i}-x_{i d}, i=$ $j+1$, where $u_{i+1}$ is the $i$ th virtual control signal. Then the time derivation of $S_{i}$ is

$$
\dot{S}_{i}=g_{i}(x)\left(x_{i+1}-u_{i+1}\right)+g_{i}(x) u_{i+1}-\frac{1}{2} S_{i}+F_{i}\left(\xi_{i}\right),
$$

where $F_{i}\left(\xi_{i}\right)=f_{i}(x)+(1 / 2) S_{i}-\dot{x}_{i d}$ is unknown; we use RBF $\mathrm{NN}$ to approximate it on a compact set $\Omega_{\xi_{i}}$,

$$
F_{i}\left(\xi_{i}\right)=W_{i}^{* T} \psi_{i}\left(\xi_{i}\right)+\Delta_{i}\left(\xi_{i}\right), \quad\left|\Delta_{i}\left(\xi_{i}\right)\right| \leq \epsilon_{i},
$$

with $\xi_{i}:=\left[x, x_{i d}, \dot{x}_{i d}\right]^{T} \in \Omega_{\xi_{i}} \subseteq \mathbb{R}^{6}$. Consider the $i$ th Lyapunov function

$$
\Gamma_{i}=\frac{1}{2} S_{i}^{2}+\frac{b_{i m}}{2 \lambda_{i}} \widetilde{\vartheta}_{i}^{2},
$$

where $\lambda_{i}$ is a positive design parameter; $\widetilde{\vartheta}_{i}=\widehat{\vartheta}_{i}-\vartheta_{i}$ with $\vartheta_{i}=\left\|W_{i}^{*}\right\|^{2} / b_{i m}$. The derivation of (36) is

$$
\begin{aligned}
\dot{\Gamma}_{i}= & g_{i}(x) S_{i}\left(x_{i+1}-u_{i+1}\right)+g_{i}(x) S_{i} u_{i+1}-\frac{1}{2} S_{i}^{2} \\
& +S_{i} F_{i}\left(\xi_{i}\right)+\frac{b_{i m}}{\lambda_{i}} \widetilde{\vartheta}_{i} \dot{\hat{\vartheta}}_{i} .
\end{aligned}
$$

Similar to (27)-(29), we have

$$
\begin{aligned}
\dot{\Gamma}_{i} \leq & g_{i}(x) S_{i}\left(x_{i+1}-u_{i+1}\right)+g_{i}(x) S_{i} u_{i+1} \\
& +\frac{b_{i m}}{2} \widehat{\vartheta}_{i} S_{i}^{2} \psi_{i}^{T}\left(\xi_{i}\right) \psi_{i}\left(\xi_{i}\right) \\
& +\frac{b_{i m}}{\lambda_{i}} \widetilde{\vartheta}_{i}\left(\dot{\hat{\vartheta}}_{i}-\frac{\lambda_{i}}{2} S_{i}^{2} \psi_{i}^{T}\left(\xi_{i}\right) \psi_{i}\left(\xi_{i}\right)\right)+\frac{1}{2}+\frac{1}{2} \epsilon_{i}^{2} .
\end{aligned}
$$

Choose the $i$ th virtual control signal

$$
u_{i+1}=-c_{i} S_{i}-\frac{1}{2} \widehat{\vartheta}_{i} S_{i} \psi_{i}^{T}\left(\xi_{i}\right) \psi_{i}\left(\xi_{i}\right)
$$

where $\widehat{\vartheta}_{i}$ is updated by

$$
\dot{\widehat{\vartheta}}_{i}=\frac{\lambda_{i}}{2} S_{i}^{2} \psi_{i}^{T}\left(\xi_{i}\right) \psi_{i}\left(\xi_{i}\right)-\lambda_{i} \sigma_{i} \widehat{\vartheta}_{i}, \quad \widehat{\vartheta}_{i}(0) \geq 0,
$$

with $c_{i}, \lambda_{i}$, and $\sigma_{i}$ positive design parameters. Substituting (39), (40), into (38), we get

$$
\begin{aligned}
\dot{\Gamma}_{i} \leq & -c_{i} b_{i} S_{i}^{2}+g_{i}(x) S_{i}\left(x_{i+1}-u_{i+1}\right)-b_{i m} \sigma_{i} \widehat{\vartheta}_{i} \widetilde{\vartheta}_{i}+\frac{1}{2} \\
& +\frac{1}{2} \epsilon_{i}^{2} .
\end{aligned}
$$

Let $u_{i+1}$ pass through the following first-order filter with time constant $\tau_{i+1}$ to obtain a new state variable $x_{(i+1) d}$,

$$
\tau_{i+1} \dot{x}_{(i+1) d}+x_{(i+1) d}=u_{i+1}, \quad x_{(i+1) d}(0)=u_{i+1}(0),
$$

Step 4. Finally, the time derivative of $S_{5}=x_{5}-x_{5 d}$ is

$$
\dot{S}_{5}=g_{5}(x) \delta_{e}-\frac{1}{2} S_{4}+F_{5}\left(\xi_{5}\right),
$$

where $F_{5}\left(\xi_{5}\right)=f_{5}(x)+(1 / 2) S_{5}-\dot{x}_{5 d}+\omega_{5}$ is unknown; we use RBF NN to approximate it on a compact set $\Omega_{\xi_{5}}$,

$$
F_{5}\left(\xi_{5}\right)=W_{5}^{* T} \psi_{5}\left(\xi_{5}\right)+\Delta_{5}\left(\xi_{5}\right), \quad\left|\Delta_{5}\left(\xi_{5}\right)\right| \leq \epsilon_{5},
$$

and $\xi_{5}=\left[x, x_{5 d}, \dot{x}_{5 d}, \omega_{5}\right]^{T} \in \Omega_{\xi_{5}} \subset \mathbb{R}^{8}$. Let

$$
\Gamma_{5}=\frac{1}{2} S_{5}^{2}+\frac{b_{5 m}}{2 \lambda_{5}} \widetilde{\vartheta}_{5}^{2},
$$

where $\lambda_{5}$ is a positive design parameter and $\widetilde{\vartheta}_{5}=\widehat{\vartheta}_{5}-\vartheta_{5}$ with $\vartheta_{5}=\left\|W_{5}^{*}\right\|^{2} / b_{5 m}$. Differentiating (45) we have

$$
\dot{\Gamma}_{5}=S_{5} g_{5}(x) \delta_{e}-\frac{1}{2} S_{5}^{2}+S_{5} F_{5}\left(\xi_{5}\right)+\frac{b_{5 m}}{\lambda_{5}} \widetilde{\vartheta}_{5} \dot{\widehat{\vartheta}}_{5} .
$$

Similar to (28), (46) can be rewritten as

$$
\begin{aligned}
\dot{\Gamma}_{5} \leq & S_{5} g_{5}(x) \delta_{e}+\frac{b_{5 m}}{2} \widehat{\vartheta}_{5} S_{5}^{2} \psi_{5}^{T}\left(\xi_{5}\right) \psi_{5}\left(\xi_{5}\right)+\frac{1}{2}+\frac{1}{2} \epsilon_{5}^{2} \\
& +\frac{b_{5 m}}{\lambda_{5}} \widetilde{\vartheta}_{5}\left(\dot{\hat{\vartheta}}_{5}-\frac{\lambda_{5}}{2} S_{5}^{2} \psi_{5}^{T}\left(\xi_{5}\right) \psi_{5}\left(\xi_{5}\right)\right) .
\end{aligned}
$$

Noting $g_{5}(x)<0$, we choose the actual control signal

$$
\delta_{e}=c_{5} S_{5}+\frac{1}{2} \widehat{\vartheta}_{5} S_{5} \psi_{5}^{T}\left(\xi_{5}\right) \psi_{5}\left(\xi_{5}\right),
$$

where $\widehat{\vartheta}_{5}$ is updated by

$$
\dot{\widehat{\vartheta}}_{5}=\frac{\lambda_{5}}{2} S_{5}^{2} \psi_{5}^{T}\left(\xi_{5}\right) \psi_{5}\left(\xi_{5}\right)-\lambda_{5} \sigma_{5} \widehat{\vartheta}_{5}, \quad \widehat{\vartheta}_{5}(0) \geq 0,
$$

with $c_{5}, \lambda_{5}$, and $\sigma_{5}$ positive design parameters. Substituting (48), (49), into (47), we arrive at

$$
\dot{\Gamma}_{5} \leq-c_{5} b_{5 m} S_{5}^{2}-b_{5 m} \sigma_{5} \widehat{\vartheta}_{5} \widetilde{\vartheta}_{5}+\frac{1}{2}+\frac{1}{2} \epsilon_{5}^{2}
$$


Remark 6. Compared with the exiting control schemes for hypersonic flight vehicle (1), the above proposed shows that, by combining DSC with the adaptive tracking controller, the design procedure can be greatly simplified and the computational burden, since only one parameter needs to be updated online in each step, can be greatly reduced.

3.4. Stability and Tracking Performance Analysis. First of all, define the filter error

$$
y_{i+1}=x_{(i+1) d}-u_{i+1}, \quad i=2,3,4 .
$$

Then, it follows that

$$
x_{i+1}-u_{i+1}=S_{i+1}+y_{i+1} .
$$

From (33) and (42),

$$
\dot{x}_{(i+1) d}=-\frac{y_{i+1}}{\tau_{i+1}} .
$$

Taking (30), (40), (51), and (53) into consideration, the time derivative of $y_{3}$ satisfies

$$
\begin{aligned}
\dot{y}_{3}= & -\frac{y_{3}}{\tau_{3}}+c_{2} \dot{S}_{2}+\frac{1}{2} \dot{\widehat{\vartheta}}_{2} S_{2} \psi_{2}^{T}\left(\xi_{2}\right) \psi_{2}\left(\xi_{2}\right) \\
& +\frac{1}{2} \widehat{\vartheta}_{2} \dot{S}_{2} \psi_{2}^{T}\left(\xi_{2}\right) \psi_{2}\left(\xi_{2}\right)+\widehat{\vartheta}_{2} S_{2} \dot{\psi}_{2}^{T}\left(\xi_{2}\right) \psi_{2}\left(\xi_{2}\right),
\end{aligned}
$$

from which we have

$$
\dot{y}_{3}=-\frac{y_{3}}{\tau_{3}}+B_{3}\left(S_{2}, S_{3}, y_{3}, \widehat{\vartheta}_{2}, x_{2 d}, \dot{x}_{2 d}, \ddot{x}_{2 d}, p_{2}\right),
$$

by the same token, we have

$$
\dot{y}_{i+1}=-\frac{y_{i+1}}{\tau_{i+1}}+B_{i+1}(\bullet),
$$

where $B_{i+1}(\bullet), i=2,3,4$, are continuous functions. From (55) and (56), the following inequalities hold:

$$
y_{i+1} \dot{y}_{i+1} \leq-\frac{y_{i+1}^{2}}{\tau_{i+1}}+\left|B_{i+1} y_{i+1}\right| .
$$

Theorem 7. Consider system (22) under Assumptions 1 and 2, with the error transformation (9), the virtual control signals (30) and (39), the control law (48), and the adaptive laws (31), (40), and (49). Then all closed-loop signals are uniformly bounded and the prescribed tracking performance (7) can be guaranteed.

Proof. Define the following Lyapunov function:

$$
\Gamma \leq \sum_{i=2}^{5} \Gamma_{i}+\sum_{i=2}^{4} \frac{1}{2} y_{i+1}^{2},
$$

where $\Gamma_{i},(i=2, \ldots, 5)$ are defined by (26), (36), and (45), respectively. The differential of the Lyapunov function $\Gamma$ is

$$
\dot{\Gamma} \leq \sum_{i=2}^{5} \dot{\Gamma}_{i}+\sum_{i=2}^{4} y_{i+1} \dot{y}_{i+1} .
$$

From (32), (41), (50), and (57) and using the following inequalities,

$$
\eta_{2} g_{2} S_{2}\left(S_{3}+y_{3}\right) \leq \eta_{2}^{2} b_{2 M}^{2} S_{2}^{2}+\frac{1}{2} S_{3}^{2}+\frac{1}{2} y_{3}^{2}-\widehat{\vartheta}_{i} \widetilde{\vartheta}_{i}
$$

$$
\begin{aligned}
& \leq-\frac{1}{2}\left(\widetilde{\vartheta}_{i}^{2}-\vartheta_{i}^{2}\right), \quad i=2, \ldots, 5, \\
S_{i} g_{i}\left(S_{i+1}+y_{i+1}\right) & \leq b_{i M}^{2} S_{i}^{2}+\frac{1}{2} S_{i+1}^{2}+\frac{1}{2} y_{i+1}^{2}, \quad i=3,4
\end{aligned}
$$

we have

$$
\begin{aligned}
\dot{\Gamma} \leq & -\eta_{2} b_{2 m} c_{2} S_{2}^{2}+\eta_{2}^{2} b_{2 M}^{2} S_{2}^{2}+\frac{1}{2} S_{3}^{2}+\frac{1}{2} y_{3}^{2} \\
& -\eta_{20} b_{2 m} \sigma_{2} \frac{1}{2}\left(\widetilde{\vartheta}_{2}^{2}-\vartheta_{2}^{2}\right)-\sum_{i=3}^{5} c_{i} b_{i m} S_{i}^{2} \\
& -\sum_{i=3}^{5} \frac{b_{i m} \sigma_{i}}{2}\left(\widetilde{\vartheta}_{i}^{2}-\vartheta_{i}^{2}\right) \\
& +\sum_{i=3}^{4}\left(b_{i M}^{2} S_{i}^{2}+\frac{1}{2} S_{i+1}^{2}+\frac{1}{2} y_{i+1}^{2}\right) \\
+ & \sum_{i=2}^{5}\left(\frac{1}{2}+\frac{1}{2} \epsilon_{i}^{2}\right)+\sum_{i=2}^{4} y_{i+1} \dot{y}_{i+1} .
\end{aligned}
$$

Define the compact sets

$$
\begin{aligned}
& \Pi_{1}:=\left\{\left(x_{2 d}, \dot{x}_{2 d}, \ddot{x}_{2 d}\right): x_{2 d}+\dot{x}_{2 d}+\ddot{x}_{2 d} \leq B_{0}\right\} \in \mathbb{R}^{3}, \\
& \Pi_{2}:=\left\{\sum_{i=2}^{5} \Gamma_{i}+\sum_{i=2}^{4} \frac{1}{2} y_{i+1}^{2} \leq \varrho\right\} \in \mathbb{R}^{14},
\end{aligned}
$$

where $\Pi_{1} \times \Pi_{2}$ is also a compact set in $\mathbb{R}^{14}$. Then, the continuous functions $B_{i+1},(i=2,3,4)$ have maximums on $\Pi_{1} \times \Pi_{2}$, say, $M_{i+1}$. Thus,

$$
y_{i+1} \dot{y}_{i+1} \leq-\frac{y_{i+1}^{2}}{\tau_{i+1}}+\frac{M_{i+1}^{2} y_{i+1}^{2}}{2 \mu}+\frac{\mu}{2}
$$

which together with (61) implies that

$$
\begin{aligned}
\dot{\Gamma} \leq & -\left(\eta_{2} b_{2 m} c_{2}-\eta_{2}^{2} b_{2 M}^{2}\right) S_{2}^{2}-\left(c_{3} b_{3 m}-\frac{1}{2}-b_{3 M}^{2}\right) S_{3}^{2} \\
& -\left(c_{4} b_{4 m}-\frac{1}{2}-b_{4 M}^{2}\right) S_{4}^{2}-\left(c_{5} b_{5 m}-\frac{1}{2}\right) S_{5}^{2} \\
& -\left(\frac{1}{\tau_{3}}-\frac{M_{3}^{2}}{2 \mu}-\frac{1}{2}\right) y_{3}^{2}-\left(\frac{1}{\tau_{4}}-\frac{M_{4}^{2}}{2 \mu}-\frac{1}{2}\right) y_{4}^{2} \\
& -\left(\frac{1}{\tau_{5}}-\frac{M_{5}^{2}}{2 \mu}-\frac{1}{2}\right) y_{5}^{2}-\frac{1}{2} \eta_{20} b_{2 m} \sigma_{2} \widetilde{\vartheta}_{2}^{2} \\
& -\sum_{i=3}^{5} \frac{b_{i m} \sigma_{i}}{2} \widetilde{\vartheta}_{i}^{2}+\sum_{i=2}^{5}\left(\frac{1}{2}+\frac{1}{2} \epsilon_{i}^{2}\right)+\frac{3 \mu}{2} \\
+ & \frac{1}{2} \eta_{20} b_{2 m} \sigma_{2} \vartheta_{2}^{2}+\sum_{i=3}^{5} \frac{b_{i m} \sigma_{i}}{2} \vartheta_{i}^{2} .
\end{aligned}
$$



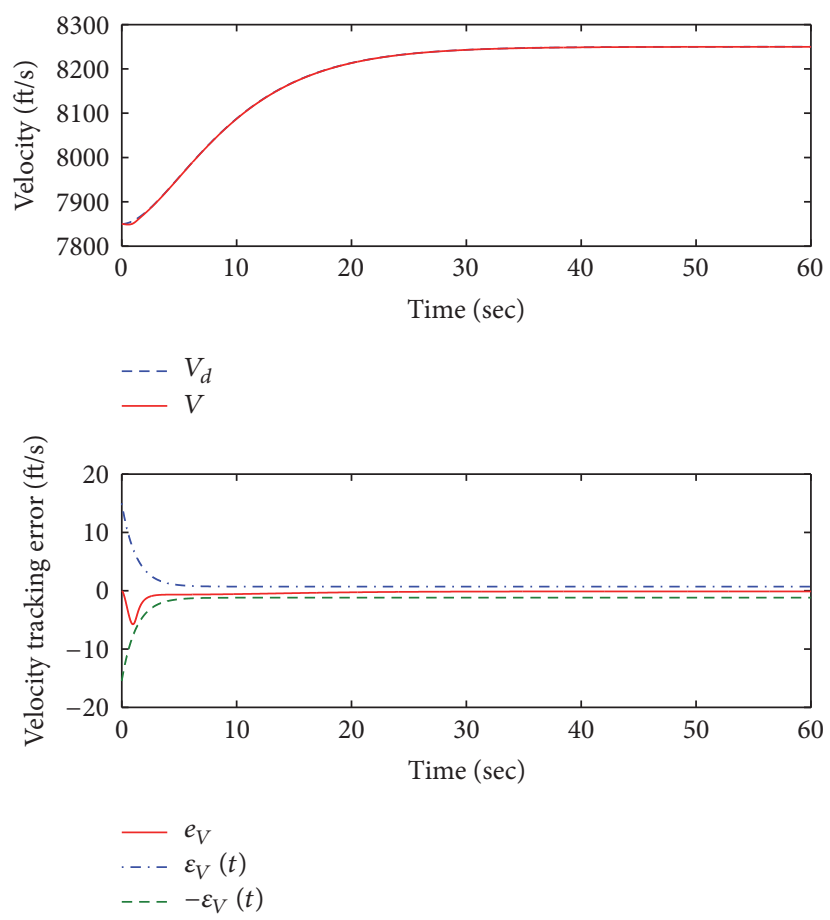

FIGURE 1: Velocity tracking performance.

Let

$$
\begin{aligned}
& \kappa_{1}=\min \left\{\left(\eta_{2} b_{2 m} c_{2}-\eta_{2}^{2} b_{2 M}^{2}\right),\left(c_{3} b_{3 m}-\frac{1}{2}-b_{3 M}^{2}\right),\left(c_{4} b_{4 m}-\frac{1}{2}-b_{4 M}^{2}\right),\left(c_{5} b_{5 m}-\frac{1}{2}\right)\right\} \\
& \kappa_{2}=\min \left\{\left(\frac{1}{\tau_{3}}-\frac{M_{3}^{2}}{2 \mu}-\frac{1}{2}\right),\left(\frac{1}{\tau_{4}}-\frac{M_{4}^{2}}{2 \mu}-\frac{1}{2}\right),\left(\frac{1}{\tau_{5}}-\frac{M_{5}^{2}}{2 \mu}-\frac{1}{2}\right)\right\}, \\
& \kappa_{3}=\min \left\{\frac{1}{2} \lambda_{2} \eta_{2} \sigma_{2}, \frac{\lambda_{i} \sigma_{i}}{2}, i=3,4,5\right\}, \\
& C=\sum_{i=2}^{5}\left(\frac{1}{2}+\frac{1}{2} \epsilon_{i}^{2}\right)+\frac{3 \mu}{2}+\eta_{2} b_{2 m} \sigma_{2} \vartheta_{2}^{2}+\sum_{i=3}^{5} \frac{b_{i m} \sigma_{i}}{2} \vartheta_{i}^{2} .
\end{aligned}
$$

Then, we have

$$
\dot{\Gamma} \leq-2 \kappa \Gamma+C,
$$

where $\kappa=\min \left\{\kappa_{1}, \kappa_{2}, \kappa_{3}\right\}$. Letting $\kappa>C / 2 \varrho$, we have $\dot{\Gamma} \leq 0$ on $\Gamma=\varrho$, which implies that if $\Gamma(0) \leq \varrho$, then $\Gamma(t) \leq \varrho, \forall t \geq 0$, and $\Gamma(t) \leq \varrho$ is an invariant set. Moreover, solving (66) yields

$$
0 \leq \Gamma(t) \leq \frac{C}{2 \kappa}+\left(\Gamma(0)-\frac{C}{2 \kappa}\right) e^{-2 \kappa t}
$$

hence, all the signals in the closed-loop system are bounded. Particularly, it follows from the boundedness of $S_{2}$ that the prescribed tracking performance (7) is guaranteed. This completes the proof.

\section{Simulation Results}

In this section, the simulation results are used to verify the effectiveness of the proposed dynamic surface control schemes. The simulation model of HFVs is taken from [18, 21]. The reference signals have been generated by filtering step reference signals through a prefilter (68), with natural frequency $\omega_{n 1}=0.5, \omega_{n 2}=0.3$, and $\zeta_{h}=0.95$. The reference signals of velocity and altitude are $400 \mathrm{ft} / \mathrm{s}$ and $1000 \mathrm{ft}$, respectively.

$$
\begin{aligned}
& \frac{x_{1 d}}{x_{1 c}}=\frac{\omega_{n_{1}}^{2}}{s^{2}+2 \zeta \omega_{n_{1}} s+\omega_{n_{1}}^{2}}, \\
& \frac{x_{2 d}}{x_{2 c}}=\frac{\omega_{n_{1}}^{2} \omega_{n_{2}}^{2}}{\left(s+\omega_{n_{1}}\right)^{2}\left(s^{2}+2 \zeta \omega_{n_{2}} s+\omega_{n_{2}}^{2}\right)} .
\end{aligned}
$$



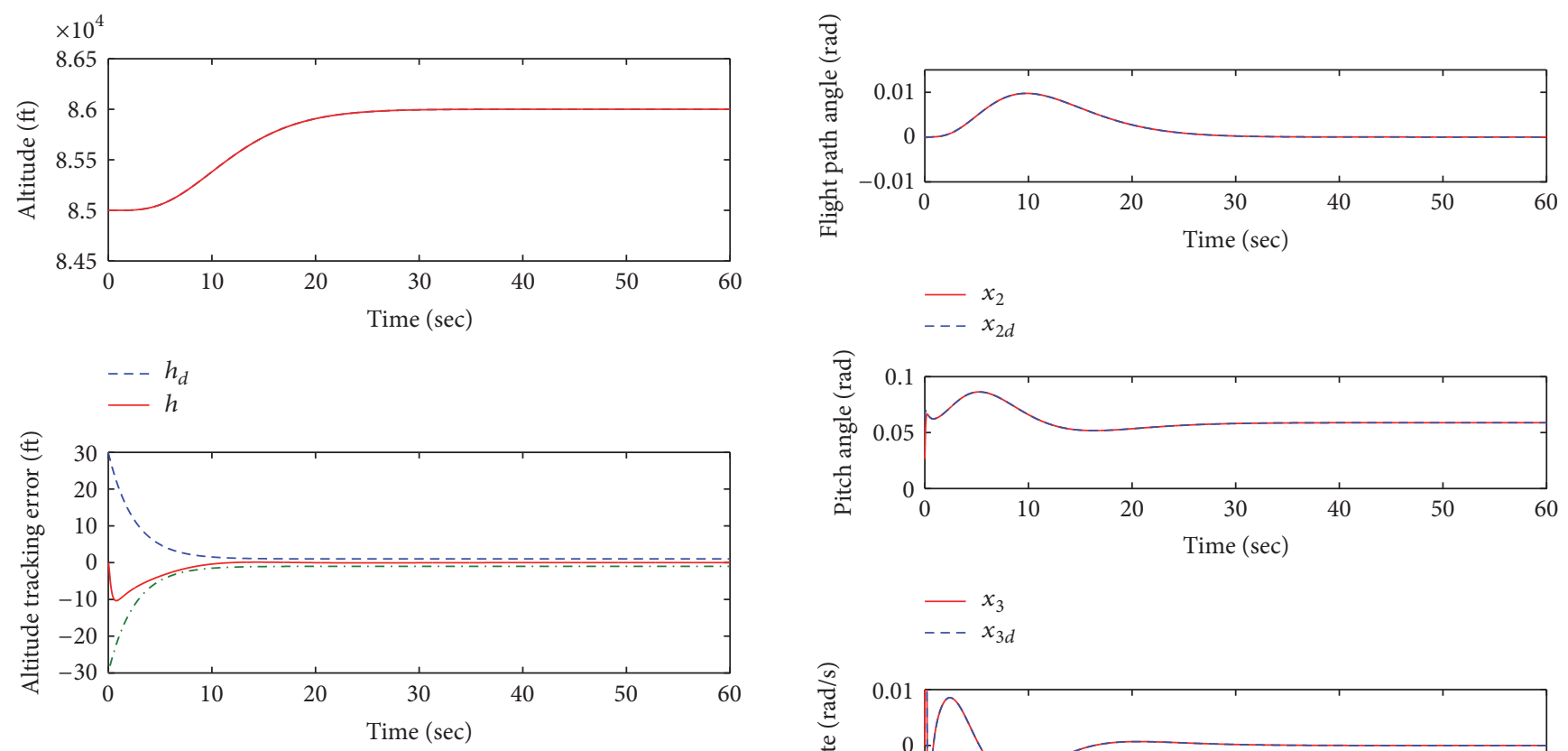

$\begin{array}{ll}- & e_{h} \\ --- & \varepsilon_{h}(t) \\ --- & -\varepsilon_{h}(t)\end{array}$

FIgURE 2: Altitude tracking performance.
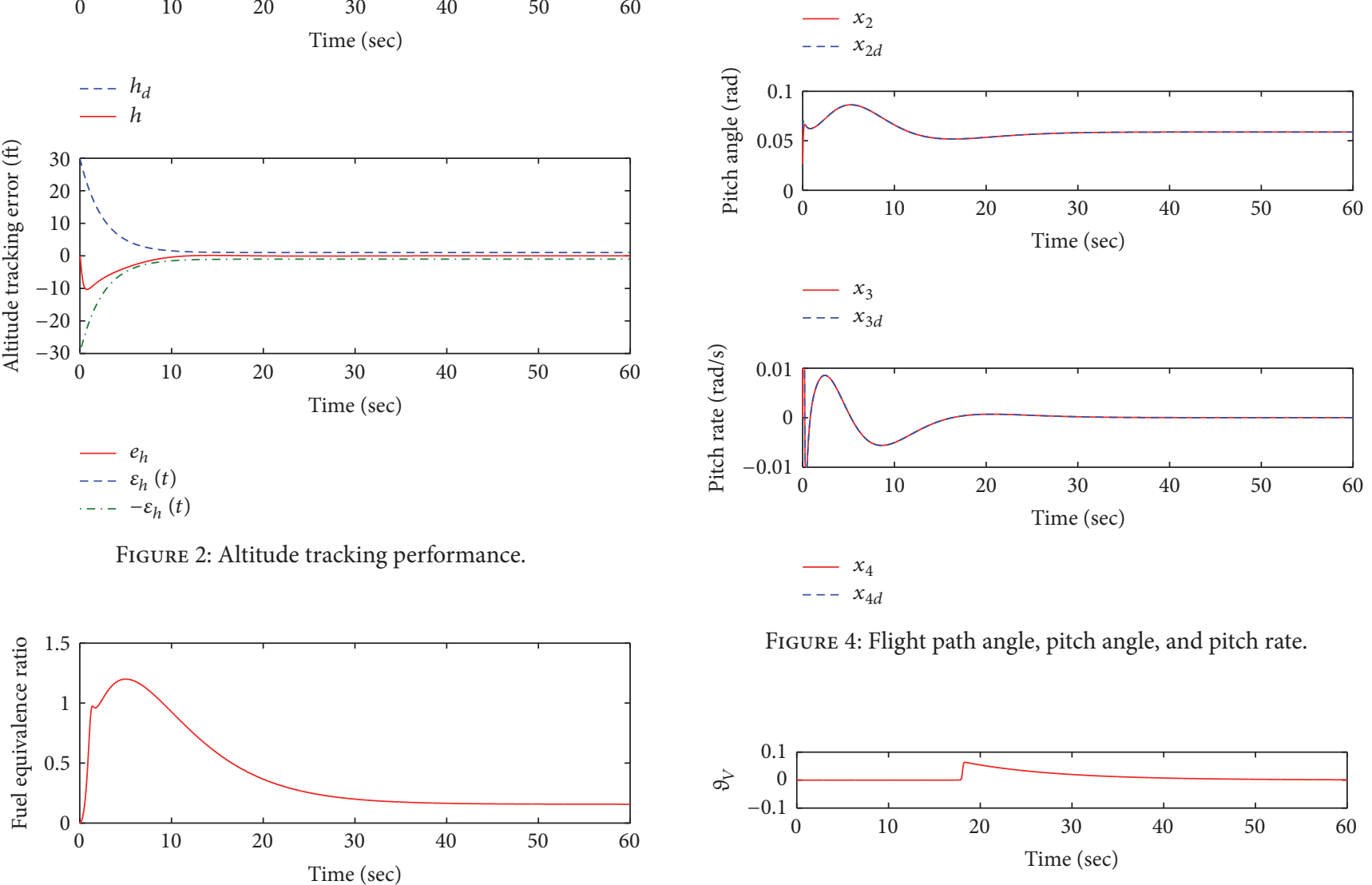

FIGURE 4: Flight path angle, pitch angle, and pitch rate.
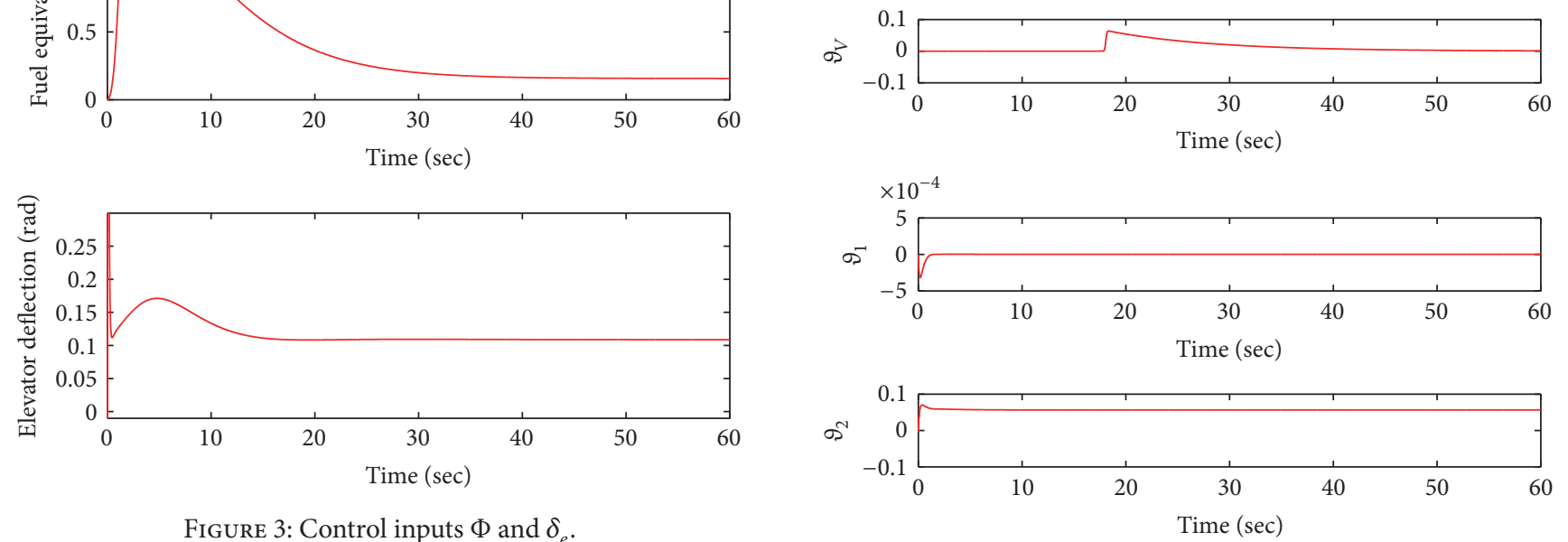

FIgure 3: Control inputs $\Phi$ and $\delta_{e}$.

In the simulation, we choose $\widehat{\vartheta}_{1}(0)=\widehat{\vartheta}_{2}(0)=\widehat{\vartheta}_{3}(0)=$ $\widehat{\vartheta}_{4}(0)=\widehat{\vartheta}_{5}(0)=0$. The design parameters are chosen as $c_{1}=$ $0.5, c_{2}=0.4, c_{3}=120, c_{4}=60, c_{5}=30, \lambda_{1}=10, \lambda_{2}=0.1$, $\lambda_{3}=0.1, \lambda_{4}=0.01, \lambda_{5}=0.01, \sigma_{1}=1, \sigma_{2}=0.1, \sigma_{3}=0.1$, $\sigma_{4}=0.1$, and $\sigma_{5}=0.1$. The initial conditions of the system (1) are $V_{0}=7850 \mathrm{ft} / \mathrm{s}, h_{0}=85000 \mathrm{ft}, \gamma_{0}=0 \mathrm{rad}, \alpha_{0}=0.0264 \mathrm{rad}$, and $q_{0}=0 \mathrm{rad} / \mathrm{s}$. The performance functions are selected as $p_{i}(t)=\left(p_{i 0}-p_{i \infty}\right) e^{-l t}+p_{i \infty}, i=1,2$, with parameters $p_{10}=$ $15, p_{1 \infty 0}=0.7, l_{1}=0.8, p_{20}=30, p_{2 \infty}=1, l_{2}=0.4$, and $m_{i}=$ $n_{i}=1$. The tracking performance is shown in Figures 1-5,

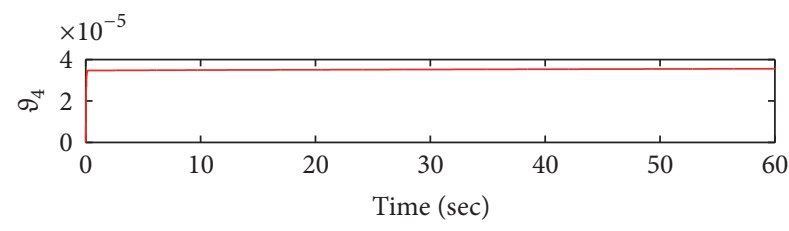

FIGURE 5: Estimation of parameters.

which indicate that the tracking performance is achieved by the proposed control scheme. 


\section{Conclusion}

This paper develops a neural network based dynamic surface controller for the HFV system in the presence of external disturbance and dynamic uncertainties. By introducing the performance and the error transformation function in controller design, the performance of tracking error can be guaranteed. Moreover, using the norm estimation approach, only one parameter needs to be updated online in each design step regardless of the plant order and input-output dimension and hence, the computational burden problem is circumvented. Numerical simulations on the COM of HFV demonstrated the effectiveness of the proposed control algorithm.

\section{Conflicts of Interest}

The authors declare that there are no conflicts of interest regarding the publication of this article.

\section{Acknowledgments}

This work was supported by the National Natural Science Foundation of China under Grant 61673101, the project of Science and Technology Development of Jilin Province, China, under Grant 20170414011GH, and Thirteenth FiveYear Science Research Plan of Jilin Province, China, under Grant JJKH20170105KJ.

\section{References}

[1] Z. Li, T. Li, B. Miao, and C. L. P. Chen, "Adaptive NN control for a class of stochastic nonlinear systems with unmodeled dynamics using DSC technique," Neurocomputing, pp. 142-150, 2015.

[2] Y. Liu, Y. Zhang, and X. Yao, "Composite antidisturbance control for a class of nonlinear stochastic systems via disturbance observer," Mathematical Problems in Engineering, vol. 2013, Article ID 281340, 2013.

[3] J. Li, W. Chen, and J.-M. Li, "Adaptive NN output-feedback decentralized stabilization for a class of large-scale stochastic nonlinear strict-feedback systems," International Journal of Robust and Nonlinear Control, vol. 21, no. 4, pp. 452-472, 2011.

[4] A. Halder and R. Bhattacharya, "Dispersion analysis in hypersonic flight during planetary entry using stochastic liouville equation," Journal of Guidance, Control, and Dynamics, vol. 34, no. 2, pp. 459-474, 2011.

[5] Z.-J. Wu, X.-J. Xie, and S.-Y. Zhang, "Adaptive backstepping controller design using stochastic small-gain theorem," Automatica. A Journal of IFAC, the International Federation of Automatic Control, vol. 43, no. 4, pp. 608-620, 2007.

[6] H. Xu, M. Mirmirani, and P. A. Ioannou, "Robust neural adaptive control of a hypersonic aircraft," in Proceedings of the AIAA Guidance, Navigation, and Control Conference and Exhibit 2003, Austin, TX, USA, August 2003.

[7] H. Xu, M. D. Mirmirani, and P. A. Ioannou, "Adaptive sliding mode control design for a hypersonic flight vehicle," Journal of Guidance, Control, and Dynamics, vol. 27, no. 5, pp. 829-838, 2004.
[8] J. T. Parker, A. Serrani, S. Yurkovich, M. A. Bolender, and D. B. Doman, "Control-oriented modeling of an air-breathing hypersonic vehicle," Journal of Guidance, Control, and Dynamics, vol. 30, no. 3, pp. 856-869, 2007.

[9] J. J. McNamara and P. P. Friedmann, "Aeroelastic and aerothermoelastic analysis in hypersonic flow: Past, present, and future," AIAA Journal, vol. 49, no. 6, pp. 1089-1122, 2011.

[10] D. O. Sigthorsson and A. Serrani, "Development of linear parameter-varying models of hypersonic air-breathing vehicles," in Proceedings of the AIAA Guidance, Navigation, and Control Conference, August 2009.

[11] J. He, R. Qi, B. Jiang, and J. Qian, "Adaptive output feedback fault-tolerant control design for hypersonic flight vehicles," Journal of the Franklin Institute. Engineering and Applied Mathematics, vol. 352, no. 5, pp. 1811-1835, 2015.

[12] Z. Gao, B. Jiang, P. Shi, J. Liu, and Y. Xu, "Passive fault-tolerant control design for near-space hypersonic vehicle dynamical system," Circuits, Systems, and Signal Processing, vol. 31, no. 2, pp. 565-581, 2012.

[13] Q. K. Shen, B. Jiang, and V. Cocquempot, "Fault-tolerant control for T-S fuzzy systems with application to near-space hypersonic vehicle with actuator faults," IEEE Transactions on Fuzzy Systems, vol. 20, no. 4, pp. 652-665, 2012.

[14] B. Jiang, Z. Gao, P. Shi, and Y. Xu, "Adaptive fault-tolerant tracking control of near-space vehicle using TakagiSugeno fuzzy models," IEEE Transactions on Fuzzy Systems, vol. 18, no. 5, pp. 1000-1007, 2010.

[15] G. Zhu and J. Liu, "Neural network-based adaptive backstepping control for hypersonic flight vehicles with prescribed tracking performance," Mathematical Problems in Engineering, Article ID 591789, Art. ID 591789, 10 pages, 2015.

[16] Y. Xu, B. Jiang, G. Tao, and Z. Gao, "Fault accommodation for near space hypersonic vehicle with actuator fault," International Journal of Innovative Computing, Information and Control, vol. 7, no. 5, pp. 2187-2200, 2011.

[17] X. Tang, G. Tao, and S. M. Joshi, "Adaptive actuator failure compensation for parametric strict feedback systems and an aircraft application," Automatica. A Journal of IFAC, the International Federation of Automatic Control, vol. 39, no. 11, pp. 1975-1982, 2003.

[18] B. Xu, X. Huang, D. Wang, and F. Sun, "Dynamic surface control of constrained hypersonic flight models with parameter estimation and actuator compensation," Asian Journal of Control, vol. 16, no. 1, pp. 162-174, 2014.

[19] X. Zhang, C.-Y. Su, Y. Lin, L. Ma, and J. Wang, "Adaptive neural network dynamic surface control for a class of timedelay nonlinear systems with hysteresis inputs and dynamic uncertainties," IEEE Transactions on Neural Networks and Learning Systems, vol. 26, no. 11, pp. 2844-2860, 2015.

[20] D. Gao, Z. Sun, and T. Du, "Dynamic surface control for hypersonic aircraft using fuzzy logic system," in Proceedings of the IEEE International Conference on Automation and Logistics (ICAL '07), pp. 2314-2319, IEEE, August 2007.

[21] W. A. Butt, "Observer based dynamic surface control of a hypersonic flight vehicle," International Journal on Smart Sensing and Intelligent Systems, vol. 6, no. 2, pp. 664-688, 2013.

[22] Z. Gao, B. Jiang, P. Shi, J. Liu, and Y. Xu, "Active fault-tolerant tracking control for near-space vehicle attitude dynamics with actuator faults," Proceedings of the Institution of Mechanical Engineers. Part I: Journal of Systems and Control Engineering, vol. 225, no. 3, pp. 413-422, 2011. 
[23] W. A. Butt, L. Yan, and A. S. Kendrick, "Adaptive integral dynamic surface control of a hypersonic flight vehicle," International Journal of Systems Science. Principles and Applications of Systems and Integration, vol. 46, no. 10, pp. 1717-1728, 2015.

[24] D. Swaroop, J. K. Hedrick, P. P. Yip, and J. C. Gerdes, "Dynamic surface control for a class of nonlinear systems," Institute of Electrical and Electronics Engineers. Transactions on Automatic Control, vol. 45, no. 10, pp. 1893-1899, 2000.

[25] X. Zhang, Z. Li, C.-Y. Su, Y. Lin, and Y. Fu, "Implementable Adaptive Inverse Control of Hysteretic Systems via Output Feedback with Application to Piezoelectric Positioning Stages," IEEE Transactions on Industrial Electronics, vol. 63, no. 9, pp. 5733-5743, 2016.

[26] R. M. Sanner and J. E. Slotine, "Gaussian networks for direct adaptive control," IEEE Transactions on Neural Networks, vol. 3, no. 6, pp. 837-863, 1992.

[27] J. Wang, Q. Zong, X. He, and H. R. Karimi, "Adaptive finite-time control for a flexible hypersonic vehicle with actuator fault," Mathematical Problems in Engineering, vol. 2013, Article ID 920796, 2013.

[28] X. Zhang, J. Wang, and L. Sun, "Robust adaptive dynamic surface control for a class of nonlinear systems preceded by complex nonlinearity," Journal of Northeast Dianli University, vol. 32, no. 3, pp. 16-20, 2012.

[29] M. Chen and S. S. Ge, "Direct adaptive neural control for a class of uncertain nonaffine nonlinear systems based on disturbance observer," IEEE Transactions on Cybernetics, vol. 43, no. 4, pp. 1213-1225, 2013.

[30] Q. Zhao and Y. Lin, "Adaptive fuzzy dynamic surface control with prespecified tracking performance for a class of nonlinear systems," Asian Journal of Control, vol. 13, no. 6, pp. 1082-1091, 2011.

[31] C. P. Bechlioulis and G. A. Rovithakis, "Adaptive control with guaranteed transient and steady state tracking error bounds for strict feedback systems," Automatica. A Journal of IFAC, the International Federation of Automatic Control, vol. 45, no. 2, pp. 532-538, 2009.

[32] C. P. Bechlioulis and G. A. Rovithakis, "Prescribed performance adaptive control for multi-input multi-output affine in the control nonlinear systems," Institute of Electrical and Electronics Engineers. Transactions on Automatic Control, vol. 55, no. 5, pp. 1220-1226, 2010. 


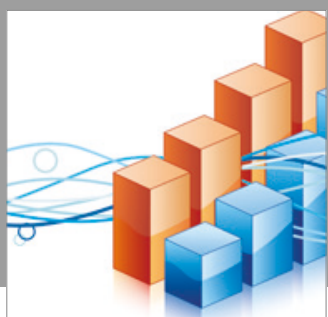

Advances in

Operations Research

vatersals

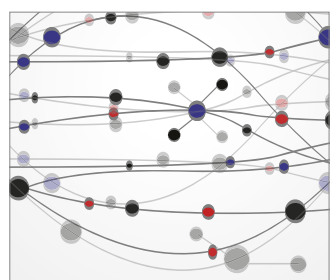

\section{The Scientific} World Journal
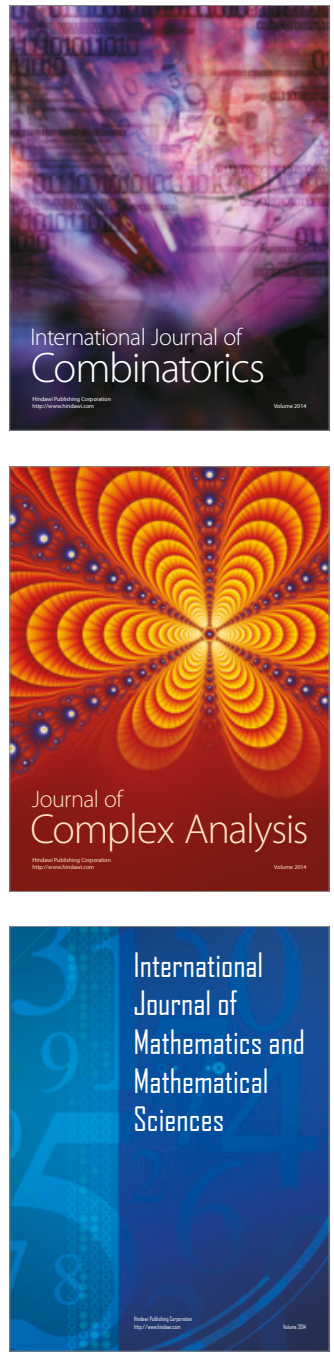
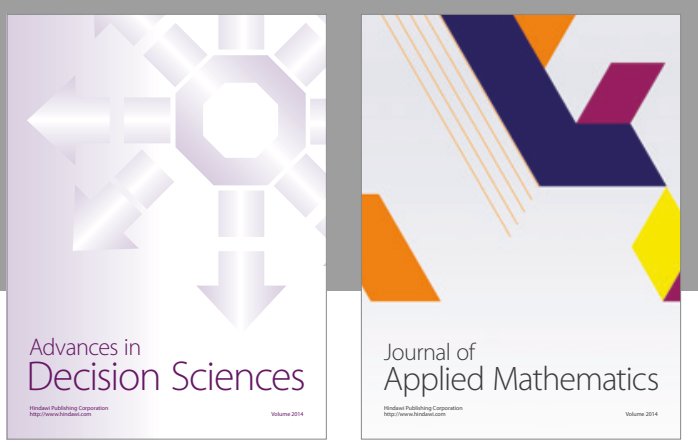

Algebra

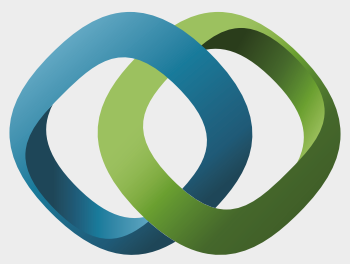

\section{Hindawi}

Submit your manuscripts at

https://www.hindawi.com
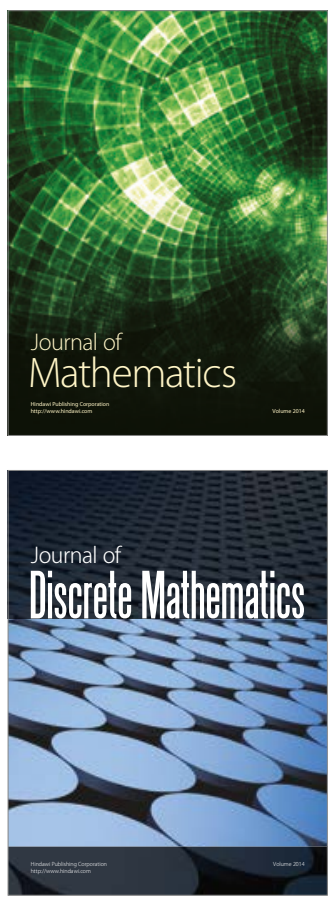

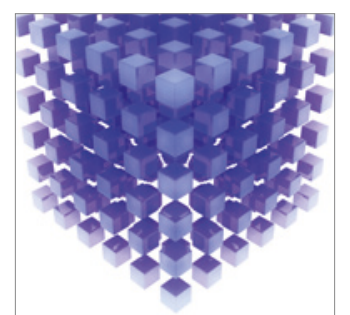

Mathematical Problems in Engineering
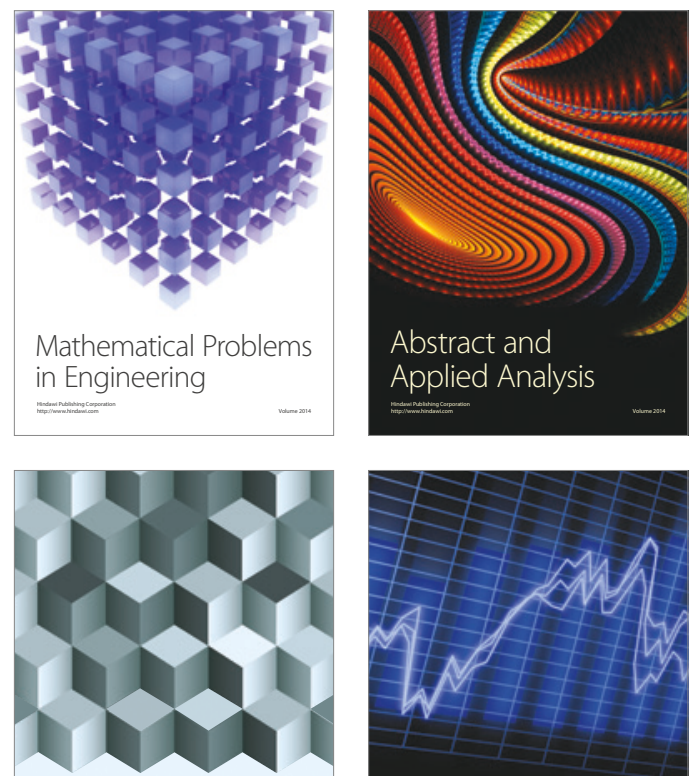

Journal of

Function Spaces

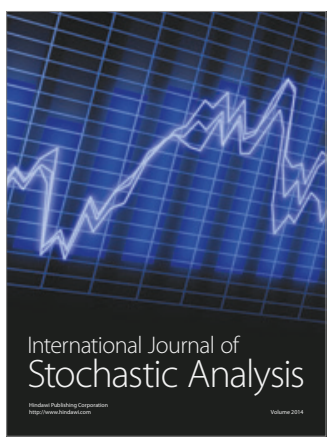

Probability and Statistics
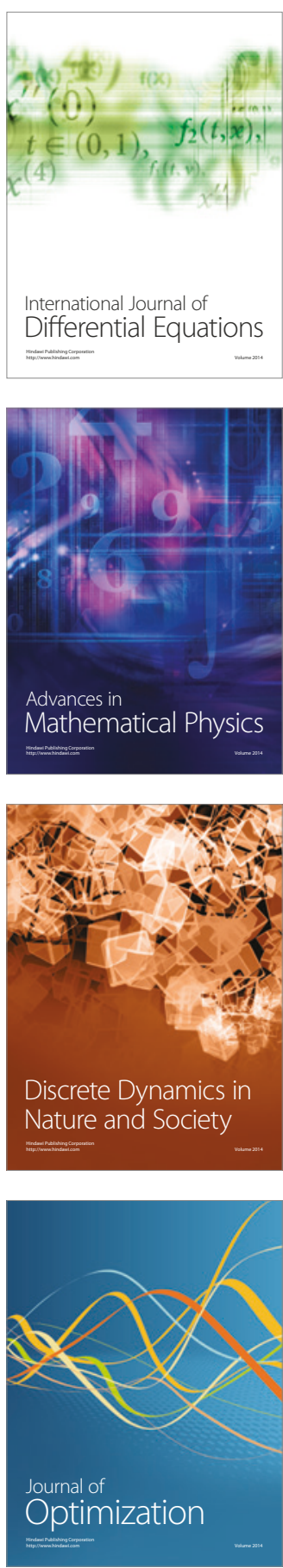